\title{
CAPIRI-IMRT: a phase II study of concurrent capecitabine and irinotecan with intensity-modulated radiation therapy for the treatment of recurrent rectal cancer
}

\author{
Gang Cai ${ }^{1+}$, Ji Zhu ${ }^{1+}$, Joshua D Palmer², Ye Xu', Weigang Hu' , Weilie Gu', Sanjun Cai ${ }^{1}$ and Zhen Zhang ${ }^{1 *}$
}

\begin{abstract}
Background: This study investigated the local effect and acute toxicity of irinotecan and capecitabine with concurrent intensity-modulated radiation therapy (IMRT) for the treatment of recurrent rectal cancer without prior pelvic irradiation.

Methods: Seventy-one patients diagnosed with recurrent rectal cancer who did not previously receive pelvic irradiation were treated in our hospital from October 2009 to July 2012. Radiotherapy was delivered to the pelvis, and IMRT of 45 Gy (1.8 Gy per fraction), followed by a boost of 10 Gy to 16 Gy (2 Gy per fraction), was delivered to the recurrent sites. The concurrent chemotherapy regimen was $50 \mathrm{mg} / \mathrm{m}^{2}$ irinotecan weekly and $625 \mathrm{mg} / \mathrm{m}^{2}$ capecitabine twice daily (Mon-Fri). Radical surgery was recommended for medically fit patients without extra-pelvic metastases. The patients were followed up every 3 months. Tumor response was evaluated using CT/MRIs according to the RECIST criteria or postoperative pathological findings. NCI-CTC 3.0 was used to score the toxicities.
\end{abstract}

Results: Forty-eight patients (67.6\%) had confirmed recurrent rectal cancer without extra pelvic metastases, and 23 patients (32.4\%) had extra pelvic metastases. Fourteen patients (19.7\%) underwent radical resections (R0) post-chemoradiation. A pathologic complete response was observed in 7 of 14 patients. A clinical complete response was observed in 4 patients (5.6\%), and a partial response was observed in 22 patients (31.0\%). Only 5 patients (7.0\%) showed progressive disease during or shortly after treatment. Of 53 symptomatic patients, clinical complete and partial symptom relief with chemoradiation was achieved in $56.6 \%$ and $32.1 \%$ of patients, respectively. Only 2 patients (2.8\%) experienced grade 4 leukopenia. The most common grade 3 toxicity was diarrhea (16 [22.5\%] patients). The median follow-up was 31 months. The cumulative local progression-free survival rate was $74.2 \%$ and $33.9 \%$ at 1 and 3 years after chemoradiation, respectively. The cumulative total survival rate was $80.1 \%$ and $36.5 \%$ at 1 and 3 years after chemoradiation, respectively.

Conclusions: This study revealed that concurrent irinotecan and capecitabine with IMRT significantly relieves local symptoms and exhibits promising efficacy with manageable toxicities in recurrent rectal cancer without prior pelvic irradiation. Improving the rate of RO resections will be investigated in a future study.

Keywords: Recurrent rectal cancer, Irinotecan, Capecitabine, Intensity-modulated radiation therapy

\footnotetext{
* Correspondence: zhenzhang6@yahoo.com

${ }^{\dagger}$ Equal contributors

'Department of Radiation Oncology, Shanghai Cancer Center, Shanghai Medical College, Fudan University, 270 Dong An Road, Shanghai, China Full list of author information is available at the end of the article
} 


\section{Introduction}

Recent advances in pretreatment radiologic evaluation, radiation and chemotherapy, and surgical techniques, such as total mesorectal excision (TME), have led to a declining incidence of locoregional recurrences for patients with primary rectal cancer [1,2]. Nevertheless, some patients still develop pelvic recurrence with or without extra pelvic metastases, especially in cases that did not receive standard initial treatment. Generally, pelvic recurrences portend a poor prognosis and are morbid, leading to troublesome symptoms, including pelvic pain, bleeding, and bowel obstruction $[3,4]$.

Currently, there is no consensus for the treatment of recurrent rectal cancer. Although radical surgery remains the only therapy with curative potential, only approximately $20-30 \%$ of patients with recurrent rectal cancer can undergo R0 resection [5]. Most patients cannot undergo curative surgery due to locally unresectable disease, medical unfitness, an unwillingness to accept the considerable associated morbidity and mortality or additional unresectable pelvic metastases. There is no survival benefit of an $\mathrm{R} 2$ resection compared with no resection [6].

Several studies have shown that radiotherapy (RT) is an effective method of treatment for recurrent rectal cancer, and a positive relationship between radiation dose and clinical outcome in recurrent rectal cancer has also been observed $[7,8]$. Therefore, there is a need to adopt new techniques, such as intensity-modified radiotherapy (IMRT), stereotactic body $\mathrm{RT}$, or proton and heavy ion RT, to safely deliver higher doses of radiation and improve local control. The combination of RT and chemotherapy with curative intent or a (neo) adjuvant setting improves the clinical outcome of patients with recurrent rectal cancer [9]. However, the optimal regimens for concurrent chemoradiation have not been clearly defined. Radiation sensitization with capecitabine has been shown to be effective for the treatment of rectal cancer. FOLFOX is currently the standard adjuvant chemotherapy; thus, most recurrent patients have previously received oxaliplatin. Both preclinical and clinical studies have revealed a synergistic effect of CPT-11 or irinotecan when combined with radiation and remarkable radiosensitizing activity of CPT-11 [10]. Several small studies have evaluated CPT11 and capecitabine combined with radiation and demonstrated that this treatment is well tolerated and effective for primary rectal cancer [11-15]. However, no previous prospective study has evaluated the efficacy and safety of irinotecan and capecitabine concurrent with IMRT for treatment of recurrent rectal cancer. Based on these considerations, we carried out a phase II trial to investigate the local effect and acute toxicity of irinotecan and capecitabine concurrent with IMRT for the treatment of recurrent rectal cancer.

\section{Materials and methods \\ Patients}

Seventy-one patients with recurrent rectal cancer were enrolled between October 2009 and November 2011. This prospective study was approved by our institutional review board (Fudan University Shanghai Cancer Center), and all patients provided informed consent.

Eligibility criteria included patients with histologically confirmed primary rectal adenocarcinoma; age between 18 and 75 years; Eastern Cooperative Oncology Group (ECOG) performance score $\leq 2$; adequate hematological, liver function, and other laboratory parameters (leucocytes $>4.0 \times 10^{9} / \mathrm{L}$, platelets $>100 \times 10^{9} / \mathrm{L}$, bilirubin $<1.5$ the upper limit of normal range (ULN); aspartate aminotransferase/alanine aminotransferase $\leq 2.5 \times \mathrm{ULN}$; and serum creatinine $<1.25 \times$ ULN).

All patients were discussed among our multidisciplinary team (MDT) before treatment and diagnosed with a pelvic recurrence either by histological confirmation or typical appearance on $\mathrm{PET} / \mathrm{CT}$, computed tomography (CT) or MRI imaging. Measurable lesions were observed in the field of RT.

Exclusion criteria were as follows: prior pelvic irradiation; prior chemotherapy with irinotecan in the last six months; any other malignancy; significant coronary or cardiac conditions; serious uncontrolled infection(s); a psychiatric disorder; and pregnancy or lack of contraception use in women with child-bearing potential.

\section{Pretreatment evaluation}

The pretreatment workup was conducted within two weeks of the treatment start date and included complete history and physical examination, digital rectal examination, colonoscopy (if possible), tumor biopsy (if possible), chest $\mathrm{CT}$, abdominal $\mathrm{CT}$ and pelvic PET/CT or $\mathrm{CT}$ or MRI, and complete laboratory tests.

\section{Radiotherapy}

RT was delivered with a linear accelerator using 6-MV photons. Every patient had a planning $\mathrm{CT}$ scan in the treatment position. IMRT planning was used for all patients based on the planning CT imaging. The target definition followed the recommendations of ICRU Report No. 83 [16]. The target volumes and nearby organs at risk were delineated on the Pinnacle $8.0 \mathrm{~m}$ planning system. The gross tumor volume (GTV) was determined by a combination of the findings on physical exam, CT, MRI, and/or PET-CT. The clinical target volume (CTV) included the GTV, internal iliac, pre-sacral and peri-rectal nodal regions, external iliac nodal region (if recurrent lesions extended into gynecologic/genitourinary structures or positive external iliac lymph nodes) and inguinal nodal region (if recurrent lesions extended to the anal verge, peri-anal skin or positive inguinal nodes). CTV was 
generated according to the RTOG anorectal contouring atlas, when available [17]. The PTV1 was generated with a $0.5-1 \mathrm{~cm}$ asymmetrical margin around the CTV. A $5 \mathrm{~mm}$ expansion was used in areas where the CTV was close to the small bowel, bladder and femoral heads, and a $10 \mathrm{~mm}$ margin was used elsewhere. The PTV2 was generated by symmetrically expanding the GTV by $2 \mathrm{~cm}$. The small bowel, bladder and femoral heads were defined as organs at risk. Inverse planning with five to seven equally spaced, coplanar IMRT fields were constructed. RT was delivered at a dose of 55-61 Gy in 3033 treatment fractions, 45 Gy in 25 fractions to PTV1 and $10-16$ Gy in 5-8 fractions to PTV2.

\section{Chemotherapy}

Irinotecan and capecitabine were delivered concurrently with RT. Starting at day 1 of RT for the duration of the irradiation (Mon-Fri), patients received capecitabine $625 \mathrm{mg} / \mathrm{m}^{2}$ orally within 30 minutes of finishing a meal, as well as weekly irinotecan at $50 \mathrm{mg} / \mathrm{m}^{2}$ for five consecutive weeks (days 1, 8, 15, 22, and 29).

\section{Maintenance chemotherapy and surgery}

After concurrent chemoradiotherapy, maintenance chemotherapy was recommended for all patients but was not included in the study protocol. Maintenance chemotherapy for the patients was individualized, with no specific recommendations.

After concurrent chemoradiotherapy, the MDT, which included a group of experienced colorectal surgeons, evaluated the clinical response of the tumor based on imaging and examined the patient to determine whether they were suitable resection candidates.

\section{Follow-up}

All patients with recurrent rectal cancer were evaluated for local responses 3 months after concurrent chemoradiation using CT, MRI, PET/CT or pathologic examination. The patients continued in serial follow up every 3 months with clinical examinations, abdominal and pelvic CTs or MRIs. Tumor response was evaluated using CT/MRIs according to the RECIST criteria or postoperative pathological findings. Adverse events were assessed at least weekly during RT using the National Cancer Institute (NCI) common toxicity criteria (NCI-CTC version 3.0).

\section{Statistical considerations}

The primary efficacy endpoint for this study was local response rate (complete or partial response). Secondary efficacy endpoints included relief of local symptoms, acute toxicity and survival.

The duration of local response and survival were analyzed using SPSS 17.0 statistical software. Survival was analyzed using the Kaplan-Meier method, and comparisons were made using log-rank tests.

\section{Results}

\section{Patient characteristics}

A total of 71 patients with recurrent rectal cancer with a median age of 53 years (range, 30-75 years) were enrolled. Enrolled patients were predominantly male ( 47 men and 24 women). Isolated pelvic locoregional recurrences occurred in 48 patients (67.6\%), whereas 23 patients (32.4\%) developed extra pelvic metastases. A total of 101 recurrent sites in all patients were included, 48 cases $(67.6 \%)$ with single sites of recurrence and 23 cases (32.4\%) with multiple recurrences. Thirty-four recurrences (33.7\%) were located in the peri-rectal region, $26(25.7 \%)$ in the presacral region, $6(5.9 \%)$ at the external iliac nodal region and $4(4.0 \%)$ at the inguinal nodal region. After concurrent chemoradiation, 56 patients $(78.9 \%)$ received 5-FU-based maintenance chemotherapy. The patient characteristics are summarized in Table 1.

At primary diagnosis, the initial staging was as follows: $6(8.5 \%)$ stage I patients, 13 (18.3\%) stage II patients, and $52(73.2 \%)$ stage III patients. A total of 45 patients (63.3\%) previously underwent low anterior resection, 25 (35.2\%) previously underwent abdominoperineal resection, and 1 (1.4\%) previously underwent local excision. In addition, 61 patients $(85.9 \%)$ previously received postoperative chemotherapy consisting of 12 cycles (range, 2-12) of 5-FU plus oxaliplatin or 6 cycles (range, 6-8) capecitabine plus oxaliplatin.

Table 1 Patient characteristics $(\mathbf{n}=71)$

\begin{tabular}{lll}
\hline Characteristics & No. & $\%$ \\
\hline Median age (range) & 53 years (30-75) & \\
\hline Gender & & \\
$\quad$ Male & 47 & 66.2 \\
$\quad$ Female & 24 & 33.8 \\
\hline Extra pelvic metastases & 48 & 67.6 \\
$\quad$ No & 23 & 32.4 \\
$\quad$ Yes & & \\
\hline Recurrent sites & 48 & 67.6 \\
Single & 23 & 32.4 \\
$\quad$ Multiple & & \\
\hline Recurrence location ( $=101)$ & 34 & 33.7 \\
Peri-rectal region & 26 & 25.7 \\
Pre-sacral region & 23 & 22.8 \\
Internal iliac nodal region & 8 & 7.9 \\
Perineum & 6 & 5.9 \\
External iliac nodal region & 4 & 4.0 \\
Inguinal nodal region & &
\end{tabular}




\section{Local response and symptom relief}

The overall local response rate was $46.5 \%$ at 3 months after concurrent chemoradiation. Complete response was observed in 11 patients (15.5\%). Fourteen patients (19.7\%) underwent radical resections (R0) post-chemoradiation. A pathologic complete response (pCR) was observed in 7 of 14 patients. Complete clinical response was observed in 4 patients (5.6\%), and a partial response was observed in 22 patients $(31.0 \%)$. Stable disease was observed in 33 patients (46.5\%). Progressive disease was observed in 5 patients $(7 \%)$ during or shortly after treatment. Overall, local control was achieved in 93\% of patients at 3 months after concurrent chemoradiation (Table 2).

Of 53 symptomatic patients, clinical complete and partial symptom relief with chemoradiation was achieved in $56.6 \%$ and $32.1 \%$ of patients, respectively (Table 2 ).

\section{Acute toxicity and dose intensity}

Table 3 demonstrates the incidence of acute toxicity during concurrent chemoradiation. Only 2 patients experienced grade 4 leukopenia. Diarrhea was the most common grade 3 toxicity. Grade 3 non-hematological toxicity included diarrhea in $22.5 \%$, radiation dermatitis in $9.9 \%$ and cystitis in $4.2 \%$ of patients. Grade 3 hand-foot skin reactions were rarely observed. Hematological toxicity of grade 3 included leukopenia in $5.6 \%$ or patients. No patients had grade 3 thrombocytopenia.

Thirty-seven patients (52.1\%) received all planned irinotecan doses (mean relative dose intensity of $75.2 \%$ ), and 41 patients (57.7\%) received all planned capecitabine doses (mean relative dose intensity $82.4 \%$ ). Sixty-five patients (91.5\%) received assigned doses of RT as scheduled.

\section{Survival}

The median follow-up for living patients after completion of chemoradiation was 31 months (range, 6-54 months). At the time of the last follow-up, clinical local control had been achieved in 42 patients (59.2\%), and 43 patients

Table 2 Summary of clinical findings

\begin{tabular}{lll}
\hline Parameter & No. of patients & $\%$ \\
\hline Response $^{*}(\mathrm{n}=71)$ & & \\
Pathologic complete response & 7 & 9.9 \\
Clinical complete response & 4 & 5.6 \\
Partial response & 22 & 31.0 \\
Stable disease & 33 & 46.5 \\
$\quad$ Progressive disease & 5 & 7.0 \\
\hline Symptom relief $(\mathrm{n}=53)$ & & \\
$\quad$ Complete relief & 30 & 56.6 \\
Partial relief & 17 & 32.1 \\
$\quad$ No relief & 6 & 11.3 \\
\hline *: 14 patients underwent radical resections (R0) post-chemoradiation. &
\end{tabular}

*: 14 patients underwent radical resections (R0) post-chemoradiation.
Table 3 Acute toxicity

\begin{tabular}{lllll}
\hline Toxicity & $\begin{array}{l}\text { Grade 1 } \\
\text { No. (\%) }\end{array}$ & $\begin{array}{l}\text { Grade 2 } \\
\text { No. (\%) }\end{array}$ & $\begin{array}{l}\text { Grade 3 } \\
\text { No. (\%) }\end{array}$ & $\begin{array}{l}\text { Grade 4 } \\
\text { No. (\%) }\end{array}$ \\
\hline $\begin{array}{lllll}\text { Hematological } \\
\text { Leukocytopenia }\end{array}$ & $9(12.7)$ & $6(8.5)$ & $4(5.6)$ & $2(2.8)$ \\
$\quad$ Thrombocytopenia & $1(1.4)$ & 0 & 0 & 0 \\
\hline Non-hematological & & & & \\
$\quad$ Diarrhea & $21(29.6)$ & $14(19.7)$ & $16(22.5)$ & 0 \\
Cystitis & $12(16.9)$ & $9(12.7)$ & $3(4.2)$ & 0 \\
Dermatitis & $8(8.5)$ & $13(18.3)$ & $7(9.9)$ & 0 \\
Vomiting & $4(5.6)$ & $3(4.2)$ & 0 & 0 \\
Hand-foot syndrome & $4(5.6)$ & $2(2.8)$ & 0 & 0 \\
\hline
\end{tabular}

developed distant metastases (60.6\%). The cumulative local progression-free survival rate was $74.2 \%$ (SE, $\pm 5.2 \%$ ) at 1 year after chemoradiation and $33.9 \%$ (SE, $\pm 6.4 \%$ ) at 3 years after chemoradiation. The median local control time was 25 months. Forty-one patients (57.5\%) died, and the cumulative total survival rate was $80.1 \%$ (SE, $\pm 4.8 \%$ ) at 1 year after chemoradiation and $36.5 \%$ (SE, $\pm 6.5 \%$ ) at 3 years after chemoradiation. The median survival time after chemoradiation was 29 months. Of the 14 patients with R0 resections, the cumulative local progression-free survival rate was $92.3 \%$ (SE, $\pm 7.4 \%$ ) at 1 year after chemoradiation and $53.3 \%$ (SE, $\pm 15.7 \%$ ) at 3 years after chemoradiation. Only 5 patients died (35.7\%), and the cumulative total survival rate was $100.0 \%(\mathrm{SE}, \pm 0 \%)$ at 1 year after chemoradiation and $59.4 \%(\mathrm{SE}, \pm 16.0 \%)$ at 3 years after chemoradiation.

\section{Discussion}

The medical literature offers little data on the optimum choice of chemotherapeutic agents to administer concurrently with radiation when treating recurrent rectal cancer. The optimal treatment for recurrent rectal cancer has not yet been defined. Patients undergoing R0 resection have the greatest survival advantage following surgery for recurrent rectal cancer [18], but many patients may not be good candidates for further surgery. In addition, approximately $50 \%$ of patients with local recurrence have concomitant metastatic disease at the time of diagnosis [19]. Multimodal treatment may offer superior outcomes in the treatment of patients with recurrent rectal cancers [9]. In addition, no prospective trial has evaluated the efficacy and safety of irinotecan and capecitabine concurrent with IMRT for the treatment of recurrent rectal cancer. This study assesses whether IMRT concurrent with irinotecan and capecitabine is effective and safe in patients with recurrent rectal cancer without prior pelvic irradiation. An overall local response rate of $46.5 \%$ and a clinical symptom relief rate of $88.7 \%$ were achieved. Only $2.8 \%$ of patients experienced grade 4 leukopenia. 
Grade 3 diarrhea, radiation dermatitis, leukopenia and cystitis were observed in $22.5 \%, 9.9 \%, 5.6 \%$ and $4.2 \%$ of patients, respectively.

Some dosimetric and clinical studies have shown that IMRT for rectal cancer can reduce the incidence of irradiated small bowel and treatment-related toxicities compared with standard 3-dimensional conformal RT (3DCRT) $[20,21]$. Therefore, a relatively higher radiation dose (55-61 Gy) using IMRT was administered to recurrent sites in our study. A number of phase I and II trials have evaluated CPT-11, capecitabine combined with radiation, and demonstrated that this treatment is well-tolerated and effective for primary rectal cancer. From these trials, the recommended irinotecan dose was $40-60 \mathrm{mg} / \mathrm{m}^{2} /$ week, and the capecitabine dose was $500-825 \mathrm{mg} / \mathrm{m}^{2}$, twice daily [11-15]. In our study, concurrent chemotherapy consisted of weekly irinotecan at $50 \mathrm{mg} / \mathrm{m}^{2}$ for five consecutive weeks (days $1,8,15,22$, and 29 ), and $625 \mathrm{mg} / \mathrm{m}^{2}$ capecitabine was administered orally (weekdays only). The regimen of IMRT concurrent with irinotecan and capecitabine is effective and safe in patients with recurrent rectal cancer.

Our results demonstrated that an overall local control rate of $93 \%$ with a local response rate of $46.5 \%$ (complete response 15.5\%) at 3 months after concurrent chemoradiation and a clinical symptom relief rate of $88.7 \%$ were achieved. These findings compare favorably with previous trials. Hu et al. [22] investigated the use of 3DCRT combined with FOLFOX4 chemotherapy in 48 patients with unresectable recurrent rectal cancer. They reported a $47.9 \%$ overall response rate in all patients. Only 1 patient achieved a complete response. Another phase II trial [23] evaluated concurrent oxaliplatin and 5-FU with RT in 102 patients with isolated locally recurrent rectal cancer revealing similar results. Complete clinical responses were observed in 13 of the 96 patients (14\%). These two trials investigated the addition of oxaliplatin to chemoradiation for the management of locally recurrent rectal cancer. Because most recurrent patients have previously received oxaliplatin after standard adjuvant chemotherapy (FOLFOX), irinotecan as a radiosensitizer in the management of recurrent rectal cancer may be a better treatment choice.

Many phase I and II trials investigating the addition of irinotecan to neoadjuvant chemoradiation for the treatment of locally advanced rectal cancer have been reported, and the pCR rate was ranged from $14 \%$ to $26 \%$ [12,13,24-26]. In our study, a pathologic complete response was observed in $9.9 \%$ (7/71) patients. Our enrolled patients were diagnosed with recurrent rectal cancer along with 32.4\% (23/71) patients with distant metastases. The proportion of patients obtaining a clinical symptom relief rate (88.7\%) was similar to that reported for some other concurrent chemoradiation protocols ( $>75 \%)$ [27].
Radical resection of recurrent tumors is crucial for longterm cure $[5,28,29]$. However, only $20 \%$ to $30 \%$ of all patients with locally recurrent rectal cancer will have a potentially curative operation. In our study, 19.7\% (14/71) of all patients and $29.2 \%(14 / 48)$ of the patients without distant metastases underwent R0 resections. Of the 14 patients with R0 resections, only 5 patients died (35.7\%), and the cumulative total survival rate was $100.0 \%(\mathrm{SE}, \pm 0 \%)$ at 1 year after chemoradiation and $59.4 \%(\mathrm{SE}, \pm 16.0 \%)$ at 3 years after chemoradiation.

Interestingly, 4 patients with a complete clinical response who did not receive surgery showed good clinical outcomes. Only 1 patient died as a result of distant metastasis, and 3 remained disease free at the time of the analysis. This strategy of close observation for clinical complete responders after preoperative CRT in locally advanced rectal cancer has been discussed [30] but requires further study.

The incidence of acute toxicity with IMRT concurrent with irinotecan and capecitabine for the treatment of recurrent rectal cancer was acceptable and manageable. Diarrhea was the most common acute toxicity. The rate of grade 3 diarrhea (22.5\%) in our study was comparable with that reported for irinotecan regimens $(11-28 \%)[12,24,31,32]$. Leukocytopenia was the most common hematologic toxicity, with grade $3-4$ recorded in $8.5 \%$ of patients. This result is similar to that reported previously [10].

The major limitation of the current study was the relatively short median follow-up of 31 months. Additionally, data regarding late toxicity are limited. Moreover, some patients who may have developed late toxicity died prematurely. The long-term efficacy and toxicity may be elucidated upon further follow-up.

In conclusion, concurrent irinotecan and capecitabine with IMRT can significantly relieve local symptoms and exhibits promising efficacy in recurrent rectal cancer without prior pelvic irradiation. Toxicity profiles were acceptable with the dosage and schedule. Improvements in the rate of $\mathrm{R} 0$ resections will be investigated in future studies. Neoadjuvant chemoradiation combining newer cytotoxic agents appears promising for the treatment of recurrent rectal cancer.

\section{Competing interests}

The authors declare that they have no competing interests.

\section{Authors' contributions}

GC, JZ and ZZ carried out study design, participated in collection and assembly of data, data analysis, manuscript writing. YX, WLG and SJC participated in study design and carried out surgery. WGH carried out radiation treatment planning. JDP revised the manuscript. All authors read and approved the final manuscript.

\section{Author details}

'Department of Radiation Oncology, Shanghai Cancer Center, Shanghai Medical College, Fudan University, 270 Dong An Road, Shanghai, China. 2Department of Radiation Oncology, Kimmel Cancer Center, Jefferson Medical College, Thomas Jefferson University, Philadelphia, PA, USA. 
Received: 1 July 2014 Accepted: 16 February 2015

Published online: 28 February 2015

\section{References}

1. van Gijn W, Marijnen CA, Nagtegaal ID, Kranenbarg EM, Putter H, Wiggers T, et al. Preoperative radiotherapy combined with total mesorectal excision for resectable rectal cancer: 12-year follow-up of the multicentre, randomised controlled TME trial. Lancet Oncol. 2011;12:575-82.

2. How P, Shihab O, Tekkis P, Brown G, Quirke P, Heald R, et al. A systematic review of cancer related patient outcomes after anterior resection and abdominoperineal excision for rectal cancer in the total mesorectal excision era. Surg Oncol. 2011;20:e149-55.

3. Garcia-Aguilar J, Cromwell JW, Marra C, Lee SH, Madoff RD, Rothenberger DA. Treatment of locally recurrent rectal cancer. Dis Colon Rectum. 2001:44:1743-8.

4. Camilleri-Brennan J, Steele RJ. The impact of recurrent rectal cancer on quality of life. Eur J Surg Oncol. 2001;27:349-53.

5. Nielsen MB, Laurberg S, Holm T. Current management of locally recurrent rectal cancer. Colorectal Dis. 2011;13:732-42.

6. Bhangu A, Ali SM, Cunningham D, Brown G, Tekkis P. Comparison of long-term survival outcome of operative vs nonoperative management of recurrent rectal cancer. Colorectal Dis. 2013;15:156-63.

7. Dresen RC, Gosens MJ, Martijn H, Nieuwenhuijzen GA, Creemers GJ, Daniels-Gooszen AW, et al. Radical resection after IORT-containing multimodality treatment is the most important determinant for outcome in patients treated for locally recurrent rectal cancer. Ann Surg Oncol. 2008;15:1937-47.

8. Mohiuddin M, Marks G, Marks J. Long-term results of reirradiation for patients with recurrent rectal carcinoma. Cancer. 2002;95:1144-50.

9. Bouchard P, Efron J. Management of recurrent rectal cancer. Ann Surg Oncol. 2010;17:1343-56.

10. Illum H. Irinotecan and radiosensitization in rectal cancer. Anticancer Drugs. 2011:22:324-9.

11. Gollins SW, Myint S, Susnerwala S, Haylock B, Wise M, Topham C, et al. Preoperative downstaging chemoradiation with concurrent irinotecan and capecitabine in MRI-defined locally advanced rectal cancer: a phase I trial (NWCOG-2). Br J Cancer. 2009:101:924-34.

12. Navarro M, Dotor E, Rivera F, Sanchez-Rovira P, Vega-Villegas ME, Cervantes A, et al. A Phase II study of preoperative radiotherapy and concomitant weekly irinotecan in combination with protracted venous infusion 5-fluorouracil, for resectable locally advanced rectal cancer. Int J Radiat Oncol Biol Phys. 2006;66:201-5.

13. Hong YS, Kim DY, Lim SB, Choi HS, Jeong SY, Jeong JY, et al. Preoperative chemoradiation with irinotecan and capecitabine in patients with locally advanced resectable rectal cancer: long-term results of a Phase II study. Int J Radiat Oncol Biol Phys. 2011;79:1171-8.

14. Hofheinz RD, von Gerstenberg-Helldorf B, Wenz F, Gnad U, Kraus-Tiefenbacher U, Muldner A, et al. Phase I trial of capecitabine and weekly irinotecan in combination with radiotherapy for neoadjuvant therapy of rectal cancer. J Clin Oncol. 2005;23:1350-7.

15. Wong SJ, Winter K, Meropol NJ, Anne PR, Kachnic L, Rashid A, et al. Radiation Therapy Oncology Group 0247: a randomized Phase II study of neoadjuvant capecitabine and irinotecan or capecitabine and oxaliplatin with concurrent radiotherapy for patients with locally advanced rectal cancer. Int J Radiat Oncol Biol Phys. 2012;82:1367-75.

16. Hodapp N. The ICRU Report 83: prescribing, recording and reporting photon-beam intensity-modulated radiation therapy (IMRT). Strahlenther Onkol. 2012;188:97-9.

17. Myerson RJ, Garofalo MC, El Naqa I, Abrams RA, Apte A, Bosch WR, et al. Elective clinical target volumes for conformal therapy in anorectal cancer: a radiation therapy oncology group consensus panel contouring atlas. Int J Radiat Oncol Biol Phys. 2009;74:824-30.

18. Bhangu A, Ali SM, Darzi A, Brown G, Tekkis P. Meta-analysis of survival based on resection margin status following surgery for recurrent rectal cancer. Colorectal Dis. 2012;14:1457-66.

19. van den Brink M, Stiggelbout AM, van den Hout WB, Kievit J, Klein Kranenbarg E, Marijnen CA, et al. Clinical nature and prognosis of locally recurrent rectal cancer after total mesorectal excision with or without preoperative radiotherapy. J Clin Oncol. 2004;22:3958-64.

20. Guerrero Urbano MT, Henrys AJ, Adams EJ, Norman AR, Bedford JL, Harrington $\mathrm{K}$, et al. Intensity-modulated radiotherapy in patients with locally advanced rectal cancer reduces volume of bowel treated to high dose levels. Int J Radiat Oncol Biol Phys. 2006;65:907-16.

21. Samuelian JM, Callister MD, Ashman JB, Young-Fadok TM, Borad MJ, Gunderson LL. Reduced acute bowel toxicity in patients treated with intensity-modulated radiotherapy for rectal cancer. Int J Radiat Oncol Biol Phys. 2012;82:1981-7.

22. Hu JB, Sun XN, Yang QC, Xu J, Wang Q, He C. Three-dimensional conformal radiotherapy combined with FOLFOX4 chemotherapy for unresectable recurrent rectal cancer. World J Gastroenterol. 2006;12:2610-4.

23. You YT, Chen JS, Wang JY, Tang R, Changchien CR, Chiang JM, et al. Concurrent chemoradiotherapy in the treatment of locally recurrent rectal cancer. Hepatogastroenterology. 2013;60:94-8.

24. Willeke F, Horisberger K, Kraus-Tiefenbacher U, Wenz F, Leitner A, Hochhaus A, et al. A phase II study of capecitabine and irinotecan in combination with concurrent pelvic radiotherapy (Caplri-RT) as neoadjuvant treatment of locally advanced rectal cancer. Br J Cancer. 2007;96:912-7.

25. Mohiuddin M, Winter K, Mitchell E, Hanna N, Yuen A, Nichols C, et al. Randomized phase II study of neoadjuvant combined-modality chemoradiation for distal rectal cancer: Radiation Therapy Oncology Group Trial 0012. J Clin Oncol. 2006;24:650-5.

26. Wahba HA, El-Hadaad HA, Roshdy S. Combination of irinotecan and 5-fluorouracil with radiation in locally advanced rectal adenocarcinoma. J Gastrointest Cancer. 2012;43:467-71.

27. Willett CG, Gunderson LL. Palliative treatment of rectal cancer: is radiotherapy alone a good option? J Gastrointest Surg. 2004;8:277-9.

28. Palmer G, Martling A, Cedermark B, Holm T. A population-based study on the management and outcome in patients with locally recurrent rectal cancer. Ann Surg Oncol. 2007;14:447-54.

29. Bakx R, Visser O, Josso J, Meijer S, Slors JF, van Lanschot JJ. Management of recurrent rectal cancer: a population based study in greater Amsterdam. World J Gastroenterol. 2008;14:6018-23.

30. Habr-Gama A, Perez RO. Non-operative management of rectal cancer after neoadjuvant chemoradiation. Br J Surg. 2009;96:125-7.

31. Voelter V, Zouhair A, Vuilleumier H, Matter M, Bouzourene H, Leyvraz S, et al. CPT-11 and concomitant hyperfractionated accelerated radiotherapy induce efficient local control in rectal cancer patients: results from a phase $\mathrm{II} . \mathrm{Br} J$ Cancer. 2006;95:710-6.

32. Mehta VK, Cho C, Ford JM, Jambalos C, Poen J, Koong A, et al. Phase II trial of preoperative $3 \mathrm{D}$ conformal radiotherapy, protracted venous infusion 5-fluorouracil, and weekly CPT-11, followed by surgery for ultrasound-staged T3 rectal cancer. Int J Radiat Oncol Biol Phys. 2003;55:132-7.

\section{Submit your next manuscript to BioMed Central and take full advantage of:}

- Convenient online submission

- Thorough peer review

- No space constraints or color figure charges

- Immediate publication on acceptance

- Inclusion in PubMed, CAS, Scopus and Google Scholar

- Research which is freely available for redistribution

Submit your manuscript at www.biomedcentral.com/submit
C BioMed Central 